\title{
ON THE CLASSIFICATION AND PROPERTIES OF NONCOMMUTATIVE DUPLICATES
}

\author{
JAVIER LÓPEZ PEÑA AND GABRIEL NAVARRO
}

\begin{abstract}
We give an explicit description of the set of all factorization structures, or twisting maps, existing between the algebras $k^{2}$ and $k^{2}$, and classify the resulting algebras up to isomorphism. In the process we relate several different approaches formerly taken to deal with this problem, filling a gap that appeared in a recent paper 8 . We also provide a counterexample to a result concerning the Hochschild (co)homology appeared in 9 .
\end{abstract}

\section{INTRODUCTION}

Interest on studying the structure of twisted tensor products of algebras comes from different sources. On the one hand, they are in one to one correspondence with factorization structures, defined in [12], on the other hand, they admit a reinterpretation as a representative for a cartesian product of spaces in the framework of noncommutative geometry ( $\mathrm{cf}[3]$, [10]). Though most of the purely algebraic properties of this construction have a very strange behaviour, which has meant a serious pitfall for most trials of systematically describing properties of twisted tensor products, the geometrical interpretation of this structure provides quite a good intuition, becoming the approach which has proved to be the most successful in the recent years.

Whilst there certainly exists a strong motivation in different areas of algebra for studying the structure of twisted tensor products, there is one basic problem concerning them which turns out to be of much more fundamental nature. Namely, the classification of all different twisted tensor products that we can obtain starting from two given algebras, say $A$ and $B$. If we are dealing with unital algebras, the equivalence between the existence of a twisted tensor product structure and the existence of a twisting map simplifies the problem into fixing a pair of vector spaces, $A$ and $B$, and finding out linear maps between $B \otimes A$ and $A \otimes B$ satisfying certain properties. In some particular situations, for instance when both $A$ and $B$ are finite dimensional, we can easily rewrite the twisting conditions in terms of the matrix elements of the linear map (once we have fixed some bases in the vector spaces), thus obtaining some polynomial equations that the component of a matrix must satisfy in order to be the representation of a twisting map in the given bases. As a consequence, we may look at the set of twisting maps between $A$ and $B$ as an affine subvariety of $M_{m \times n}(k)$, where $m$ is the dimension of $A$ and $n$ is the dimension

This research has been partially supported by the projects MTM2004-08125, MTM2004-01406 and FQM-266 (Junta de Andalucía Research Group). First author is partially supported by Spanish MECFPU grant AP2003-4340. 
of $B$. The classification of the twisting maps is therefore equivalent to the description of this algebraic variety. Some steps on describing the variety of twisting maps has been given by Cibils in 8 .

The second obvious problem that we face when trying to classify the twisted tensor products between two algebras is the fact that different twisting maps may give rise to isomorphic algebra structure. In terms of the variety of twisting maps, this problem boils down to study a quotient variety of our original one. Unfortunately, so far no groundwork that can simplify the isomorphism problem is known, and we are bond to deal with each case separately.

Though several attempts to describe the structure and behavior of twisted tensor products of algebras has been taken, the inherent difficulty of the task has caused a number of flaws in different papers on the topic. Our purpose in this work is to clarify, through the exhaustive description of the space of twisting maps between two particular algebras and the orbitspace of isomorphism classes associated to them, some points that have recently appeared in the literature under an incorrect form. In particular, we fix a subtle mistake in Cibils' classification of noncommutative duplicates. We also give a counterexample to several results given by J.A. Guccione and J.J. Guccione in [9], concerning Hochschild homology of twisted tensor products.

It is worth noting that our example stress the fact that the homological properties of a twisted tensor product is usually not well behaved with respect to the same properties in the factors, even if we impose some conditions on the twisting map. However, if we impose some stronger conditions on the involved algebras, it is still possible to recover some regular behaviour. In particular, Koszulity of the algebras is enough to guarantee Koszulity of the twisted tensor product, giving rise to a Künneth formula describing the homology of the product algebra in terms of the homology of the factors. Further details in this matter can be found on [11].

\section{Preliminaries}

Along the present work, $k$ will stand for a ground field, which we will require in some situations, explicitly mentioned in the text, to be of characteristic different from 2. All algebras will be supposed to be associative, unital, $k$-algebras. The term linear will always mean $k$-linear, and the unadorned tensor product $\otimes$ will stand for the usual tensor product over $k$. For an algebra $A$ we will write $\mu_{A}$ to denote the product in $A$ and $u_{A}: k \rightarrow A$ its unit. We will also identify every object with the identity map defined on it, so that $A \otimes f$ will mean $\operatorname{Id}_{A} \otimes f$.

The notion of twisted tensor product has been independently discovered a number of times. Some of the earliest papers giving the definition that we shall use are [3], [4]. Let $A, B$ be two algebras, and $\tau: B \otimes A \rightarrow A \otimes B$ a linear map. We say that $\tau$ is a twisting 
map if it satisfies the following conditions:

$$
\begin{gathered}
\tau(b \otimes 1)=1 \otimes b, \quad \tau(1 \otimes a)=a \otimes 1, \quad \forall a \in A, b \in B, \\
\tau \circ\left(B \otimes \mu_{A}\right)=\left(\mu_{A} \otimes B\right) \circ(A \otimes \tau) \circ(\tau \otimes A), \\
\tau \circ\left(\mu_{B} \otimes A\right)=\left(A \otimes \mu_{B}\right) \circ(\tau \otimes B) \circ(B \otimes \tau) .
\end{gathered}
$$

If $\tau$ is a twisting map, then the map defined on $A \otimes B$ by $\mu_{\tau}:=\left(\mu_{A} \otimes \mu_{B}\right) \circ(A \otimes \tau \otimes B)$, is an associative multiplication and $1 \otimes 1$ is the unit. The algebra having $A \otimes B$ as underlying vector space and $\mu_{\tau}$ as a product is denoted by $A \otimes_{\tau} B$ and is called the twisted tensor product of $A$ and $B$ (with respect to the twisting map $\tau$ ). For unital algebras, the existence of a twisting map is equivalent to the existence of an algebra structure on $A \otimes B$ which is compatible with the canonical inclusions of $A$ and $B$, and thus twisting maps are in one to one correspondence with the so-called factorization structures introduced in [12]. From a categorical point of view, a twisted tensor product is a particular situation of the theory of distributive laws introduced by J. Beck in [1].

Very little is known about the classification of the existing twisting maps between two given algebras. Even in the simplest cases, this turns out to be a very difficult problem to tackle. In [8], C. Cibils proposed a method for describing all the twisting maps between $A$ and $B$, being $A=k^{n}$ the algebra of functions over an $n$-points set, and $B=k^{2}$ the two-points algebra. The resulting twisted tensor product algebras, which are dubbed noncommutative duplicates can be realized up to some extent as a sort of Ore extensions associated to the quotient algebra $k[x] /\left(x^{2}-x\right)$. For the sake of completeness, we sketch the procedure followed by Cibils for obtaining the classification of the noncommutative duplicates.

Proposition 1.1. The set of twisting maps between $A$ and $k^{2}$ (also called the set of 2-interlacings of $A$ ) is in one to one correspondence with the set $Y_{A}$ of couples $(f, \delta)$ with $f \in$ End $A$ an algebra endomorphism and $\delta: A \rightarrow A$ an idempotent $f$-twisted derivation such that

$$
f=f^{2}+\delta f+f \delta
$$

Every algebra endomorphism $f$ of the algebra $A=k^{n}$ may be given in terms of a set map $\varphi$, to which we can associate a one-valued quiver with $n$ vertices. To this quiver, using the derivation $\delta$ we may assign a coloration satisfying certain conditions. Conversely, every one valued quiver which admits a coloration satisfying those properties give rise to an algebra endomorphism and a derivation as in the former proposition, and thus to a twisting map, and so there is a one to one correspondence between the set of 2-interlacings of $k^{n}$ and the set of coloured one valued quivers with $n$ vertices.

Using this equivalence, Cibils gives a classification of all the noncommutative duplicates of the algebras $k^{n}$, and computes their Hochschild (co)homology using the techniques developed in [7]. More concretely, the following results are used in order to compute the Hochschild cohomology: 
Theorem $1.2([8])$. Let $Q$ be a connected quiver which is not a crown, and let us denote by $(K Q)_{2}$ the quotient of the path algebra $k Q$ by the two sided ideal $\left(Q_{\geq 2}\right)$ generated by the paths of length 2 , then we have:

(1) $\operatorname{dim}_{k} H H^{0}\left((k Q)_{2}\right)=\#\left(Q_{1} / / Q_{0}\right)+1$,

(2) $\operatorname{dim}_{k} H H^{1}\left((k Q)_{2}\right)=\#\left(Q_{1} / / Q_{1}\right)-\#\left(Q_{0}\right)+1$,

(3) $\operatorname{dim}_{k} H H^{n}\left((k Q)_{2}\right)=\#\left(Q_{n} / / Q_{1}\right)-\#\left(Q_{n-1} / / Q_{0}\right)$ for all $n \geq 2$.

where for two sets of paths $X$ and $Y$, by $X / / Y$ we denote the set of parallel paths, that is, the set of couples $(x, y) \in X \times Y$ where $x$ and $y$ have the same source and target. Proposition $1.3([7])$. Let $Q$ be a $c$-crown, with $c \geq 2$, then the center of $(k Q)_{2}$ is one-dimensional. If the characteristic of $k$ is different from 2 , for any $n$ which is an even multiple of $c$ we have

$$
\operatorname{dim}_{k} H H^{n}\left((k Q)_{2}\right)=\operatorname{dim}_{k} H H^{n+1}\left((k Q)_{2}\right)=1 .
$$

The cohomology vanishes in all other degrees.

These two results have an important consequence (see Corollary 3.2 of [7]):

Corollary 1.4. Let $Q$ be a connected quiver which is not a crown, then the graded cohomology $\mathrm{HH}^{\bullet}\left((k Q)_{2}\right)$ is finite dimensional if, and only if, $Q$ has no oriented cycles.

\section{The space of twisting Maps}

Let us consider the algebras $A$ and $B$ both isomorphic to $k\left[\mathbb{Z}_{2}\right]$, the ring algebra of the cyclic group $\mathbb{Z}_{2}$, and let us fix $\left\langle 1_{A}, a\right\rangle$ basis of $A$ and $\left\langle 1_{B}, b\right\rangle$ basis of $B$, satisfying $a^{2}=1_{A}$ and $b^{2}=1_{B}$. Then the set

$$
\left\langle 1_{A} \otimes 1_{B}, 1_{A} \otimes b, a \otimes 1_{B}, a \otimes b\right\rangle
$$

is a basis of $A \otimes B$, and

$$
\left\langle 1_{B} \otimes 1_{A}, b \otimes 1_{A}, 1_{B} \otimes a, b \otimes a\right\rangle
$$

is a basis of $B \otimes A$.

The choice of these bases simplifies the required computations for finding out all the twisting maps $\tau: B \otimes A \rightarrow A \otimes B$, since the unitality conditions on $\tau$ forces us to take

$$
\tau(1 \otimes 1)=1 \otimes 1, \tau(1 \otimes a)=a \otimes 1, \tau(b \otimes 1)=1 \otimes b,
$$

so in order to give a twisting map between $A$ and $B$ it is enough to give a value for $\tau(b \otimes a)$ and check that it satisfies the required compatibility conditions with respect to multiplications in $A$ and $B$. In [2], an explicit approach to this problem is performed, obtaining that any twisting map is one of the following list:

(a) If $\operatorname{char}(k)=2$, then:

(i) $\tau(b \otimes a)=\alpha\left(1_{A} \otimes 1_{B}\right)+(a \otimes b)$, where $\alpha \in k$.

(ii) $\tau(b \otimes a)=\alpha\left(1_{A} \otimes 1_{B}\right)+\alpha\left(1_{A} \otimes b\right)+\alpha\left(a \otimes 1_{B}\right)+(\alpha+1)(a \otimes b)$, where $\alpha \in k$.

(b) If $\operatorname{char}(k) \neq 2$, then:

(i) $\tau(b \otimes a)=(a \otimes b)$.

(ii) $\tau(b \otimes a)=-\left(1_{A} \otimes 1_{B}\right)+\alpha(a \otimes b)$, where $\alpha \in k$. 
(iii) $\tau(b \otimes a)=-\left(1_{A} \otimes 1_{B}\right)+\left(1_{A} \otimes b\right)+\left(a \otimes 1_{B}\right)$.

(iv) $\tau(b \otimes a)=\left(1_{A} \otimes 1_{B}\right)-\left(1_{A} \otimes b\right)+\left(a \otimes 1_{B}\right)$.

(v) $\tau(b \otimes a)=\left(1_{A} \otimes 1_{B}\right)+\alpha\left(1_{A} \otimes b\right)-\left(a \otimes 1_{B}\right)$.

(vi) $\tau(b \otimes a)=-\left(1_{A} \otimes 1_{B}\right)-\left(1_{A} \otimes b\right)-\left(a \otimes 1_{B}\right)$.

The space of twisting maps over these particular algebras may also be computed by means of certain coloured quivers, which are associated to twisting maps following the procedure developed in [8], as summarized at the end of Section 1

In our situation, the algebra maps $f: k^{2} \rightarrow k^{2}$ are all given as the lifting of the set maps $\varphi:\{a, b\} \rightarrow\{a, b\}$ (see [8] for this correspondence), thus obtaining the four possible algebra maps given in generators by:

- $f_{1}(a)=a$ and $f_{1}(b)=b$.

- $f_{2}(a)=b$ and $f_{2}(b)=a$.

- $f_{3}(a)=a+b$ and $f_{3}(b)=0$.

- $f_{4}(a)=0$ and $f_{4}(b)=a+b$.

Associated to these maps, we have the following quivers (where $Q_{i}$ stands for the quiver associated to the algebra map $f_{i}$ ):

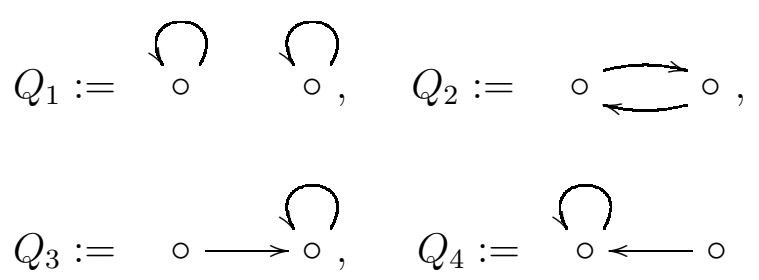

Now, the colorations attached to these quivers are given by:

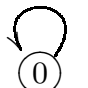<smiles>c1ccccc1</smiles>

$\left(i i^{\prime}\right) \underset{\longrightarrow}{\longrightarrow}$ where $\beta=-1-\alpha$.

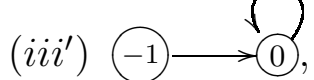<smiles>[Ge]c1c[c-]ccc1</smiles><smiles>[C+]c1ccccc1</smiles>

Here we may observe that the twisting map $(i)$ corresponds to the coloured quiver $\left(i^{\prime}\right)$, the one-parameter family of maps $(i i)$ is associated to the quivers $\left(i i^{\prime}\right)$ when we vary the coloration, and the twisting maps $(i i i),(i v),(v)$ and $(v i)$ correspond to the given colorations of $\left(i i i^{\prime}\right)$.

Remark. As a consequence of all this, the set of twisting maps gives rise to a variety consisting in five isolated points, which correspond to the twisting maps (i) and (iii)-(vi), plus a $k$-line, associated to the one-parameter family of maps described in $(i i)$.

\section{The ISOMORPhism ClASses OF THE TWISTED ALGEBRAS}

In the former section we described the set of all twisting maps between $k^{2}$ and $k^{2}$ but, as we mentioned earlier, different twisting maps could give rise to isomorphic algebras. In this section we will describe the algebras associated to the twisting maps that we 
obtained in the previous subsection, describing the different isomorphism classes and giving a description of the orbitspace in the corresponding variety of twisting maps.

In [2], a description of these algebras by means of generators and relations is given, in particular mentioning that the algebras obtained from the (non-invertible) twisting maps (iii)-(vi) are all isomorphic to

$$
k\left\langle a, b \mid a^{2}=b^{2}=1, b a=a+b+1\right\rangle .
$$

A different, but equivalent, description may be given following [8], where it is shown that the algebras associated to the four non-invertible twisting maps are all isomorphic to the path algebra of the quiver

$$
\widetilde{Q}:=\quad \circ \stackrel{\circ}{\longrightarrow} \circ
$$

This means that four out of the five isolated points in our variety provides the same point in the orbitspace. For the remaining isolated point, which is the one corresponding to the flip map, i.e. $(i)$, the corresponding algebra is just the usual tensor product:

$$
k \mathbb{Z}_{2} \otimes k \mathbb{Z}_{2} \cong k\left\langle a, b \mid a^{2}=b^{2}=1, b a=a b\right\rangle .
$$

Again, this algebra may be described as the path algebra of the quiver

$$
\begin{array}{ll}
0 & 0 \\
0 & 0
\end{array}
$$

This algebra is clearly non-isomorphic to the former one, since it is commutative, and thus it gives a new point in the orbitspace.

Henceforth, the only remaining case is the one-parameter family of twisting maps described in $(i i)$. The family of algebras obtained out of these twisting maps is described in 2] in terms of generators and relations, obtaining the family

$$
A_{q}:=k\left\langle a, b \mid a^{2}=b^{2}=1, a b+b a=q\right\rangle, \quad \text { where } q \in k .
$$

The authors of [2] are not concerned by the number of different isomorphism classes of algebras which are obtained according to different values of the parameter. On the other hand, according to [8, Theorem 4.4], all these algebras should be isomorphic to the quotient of the path algebra of the so-called round-trip quiver

$$
Q:=0 \longrightarrow 0
$$

modulo the ideal generated by the set $Q_{\geq 2}$ of paths of length greater than one. In other words, the obtained algebra would not depend on the coloration. Unfortunately, the proof contains a slight mistake. Within this one-parameter family of algebras we can find two different kinds of algebras:

- If we take $q \neq \pm 2$, then the algebra map

$$
k\langle a, b\rangle \longrightarrow \mathcal{M}_{2}(k)
$$


defined by

$$
a \longmapsto\left(\begin{array}{cc}
1 & 0 \\
0 & -1
\end{array}\right), \quad b \longmapsto\left(\begin{array}{cc}
\frac{q}{2} & \frac{2-q}{4} \\
\frac{2+q}{4} & -\frac{q}{2}
\end{array}\right)
$$

provides an isomorphism of algebras between the algebra $A_{q}$ and the $2 \times 2$ matrix ring $\mathcal{M}_{2}(k)$.

- If $q \in\{2,-2\}$, the algebra map $f: A_{-2} \rightarrow A_{2}$ defined by

$$
\begin{gathered}
f\left(1_{A} \otimes 1_{B}\right):=\left(1_{A} \otimes 1_{B}\right), \quad f\left(1_{A} \otimes b\right):=\left(a \otimes 1_{B}\right), \\
f\left(a \otimes 1_{B}\right):=\left(1_{A} \otimes b\right)-2\left(a \otimes 1_{B}\right), \quad f(a \otimes b):=-(a \otimes b)
\end{gathered}
$$

is an isomorphism.

Now, consider $R:=k Q /\left(Q_{\geq 2}\right)$ the quotient of the path algebra of the roundtrip quiver modulo the ideal generated by $Q_{\geq 2}$. We may explicitly describe $R$ as the algebra having a basis consisting in the four elements $e, f, x, y$ such that the multiplication is given by the following table:

\begin{tabular}{c|cccc} 
& $e$ & $f$ & $x$ & $y$ \\
\hline$e$ & $e$ & 0 & 0 & $y$ \\
$f$ & 0 & $f$ & $x$ & 0 \\
$x$ & $x$ & 0 & 0 & 0 \\
$y$ & 0 & $y$ & 0 & 0
\end{tabular}

Considering the algebra map $\phi: R \rightarrow A_{-2}$ defined by:

$$
\begin{gathered}
\phi(e):=1 / 2\left(\left(1_{A} \otimes 1_{B}\right)-\left(a \otimes 1_{B}\right)\right), \\
\phi(f):=1 / 2\left(\left(1_{A} \otimes 1_{B}\right)+\left(a \otimes 1_{B}\right)\right), \\
\phi(x):=1 / 4\left(\left(1_{A} \otimes 1_{B}\right)+\left(1_{A} \otimes b\right)+\left(a \otimes 1_{B}\right)+(a \otimes b)\right), \\
\phi(y):=1 / 4\left(\left(1_{A} \otimes 1_{B}\right)-\left(1_{A} \otimes b\right)-\left(a \otimes 1_{B}\right)+(a \otimes b)\right),
\end{gathered}
$$

we have $\phi$ is an algebra isomorphism between $A_{-2}$ and $R$, obtaining that both $A_{2}$ and $A_{-2}$ are isomorphic to the algebra $R$.

Finally, the line corresponding to our one-parameter family of twisting maps corresponds to two points in the orbit space, one open orbit corresponding to the matrix ring, plus one more point in the closure of this orbit, corresponding to the quotient of the path algebra of the round-trip quiver. From the point of view of deformation theory, this means that the matrix algebra, realized as a twisted tensor product, admits a deformation to the algebra $k Q /\left(Q_{\geq 2}\right)$.

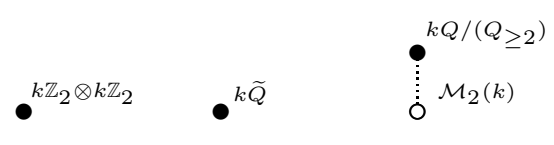

Figure 1. The orbit space

Summarizing, we have proven the following result: 
Proposition 3.1. Let $k$ be a field with $\operatorname{char}(k) \neq 2$. Let $A \cong B \cong k^{2}$, and let $\tau$ : $B \otimes A \rightarrow A \otimes B$ be a twisting map, then the twisted tensor product algebra $R: A \otimes_{\tau} B$ must be isomorphic to one of the following algebras:

(I) $k^{4}$, or equivalently, the path algebra of the quiver

$\begin{array}{ll}0 & 0 \\ 0 & 0\end{array}$

(IIa) The algebra of matrices $\mathcal{M}_{2}(k)$.

(IIb) The quotient $k Q /\left(Q_{\geq 2}\right)$ of the path algebra $k Q$ of the round-trip quiver

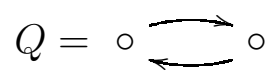

modulo the ideal generated by the set $Q_{\geq 2}$ of paths of length greater than one.

(III) The path algebra $k \widetilde{Q}$ of the quiver

$$
\widetilde{Q}=\circ \stackrel{\circ}{\longrightarrow} \circ
$$

Remark. As we mentioned above, the classification given by Cibils for noncommutative duplicates of set algebras in [8], is almost complete, with the only exception being given by Theorem 4.4, dealing with the connected components of the (coloured) quivers that are precisely the round-trip quiver.

Next we consider the formalism developed by Cibils for a two-fold purpose, namely to highlight where the slight mistake in his proof have been done and to obtain a characteristic free classification of the isomorphism classes. We have communicated to Cibils the complete previous classification we have obtained, then he provided us the precise localisation of the error in [8].

Following the same notation as Cibils does in [8], the algebra structure of $A \otimes k[X] /\left(X^{2}-\right.$ $X)$ is determined by the products

$$
X a=\tau(X \otimes a)=\delta(a)+f(a) X
$$

for each $a \in A$, where $(\delta, f)$ is the pair of the derivation and the endomorphism associated to the twisting map $\tau$, see [8, Proposition 2.10].

In our particular situation, that is, when we deal with the round-trip quiver, the algebra endomorphism is given by

$$
f(u)=v, f(v)=u
$$

whilst the derivation is given by

$$
\delta(u)=a_{v} v-a_{u} u, \delta(v)=a_{u} u-a_{v} v
$$

being $a_{u}$ and $a_{v}$ some parameters in $k$ and, $u$ and $v$ the primitive orthogonal idempotent elements of $k^{2}=k\{u, v\}$ (cf. [8, Lemma 3.3]). Applying formula (3.4) to this particular 
situation we have:

$$
\begin{gathered}
X u=-a_{u} u+a_{u} v+v X \\
X v=a_{u} u-a_{u} v+u X .
\end{gathered}
$$

Remember that in order to get a well-defined, associative structure, it is necessary and sufficient to have $a_{u}+a_{v}+1=0$, as mentioned in [8, Theorem 3.14]. Using this, the multiplication of the resulting algebra may be summarized in the following table:

\begin{tabular}{c|cccc} 
& $u$ & $u X$ & $v$ & $v X$ \\
\hline$u$ & $u$ & $u X$ & 0 & 0 \\
$u X$ & $-a_{u} u$ & $-a_{u} u X$ & $a_{u} u+u X$ & $-a_{v} u X$ \\
$v$ & 0 & 0 & $v$ & $v X$ \\
$v X$ & $a_{v} v+v X$ & $-a_{u} v X$ & $-a_{v} v$ & $-a_{v} v X$
\end{tabular}

Now, observe that we have

$$
\begin{aligned}
& (v X u)(u X v)=(v X)(u X) v=a_{u} a_{v} v \\
& (u X v)(v X u)=(u X)(v X) u=a_{u} a_{v} u
\end{aligned}
$$

and this products are zero if, and only if, $a_{u} a_{v}=0$, a condition which is equivalent to have $a_{u}=0$ and $a_{v}=-1$, or $a_{u}=-1$ and $a_{v}=0$. In this two cases we may carry on with the proof of [8, Theorem 4.4], obtaining the isomorphism with the quotient of the path algebra of the round-trip quiver, as Cibils states (in our classification these algebras correspond to $A_{2}$ and $A_{-2}$ ).

However, if the product $a_{u} a_{v}$ is non-zero, that is, if neither $a_{u}$ nor $a_{v}$ are 0 , then the map $\psi: k Q_{f} \rightarrow k\{u, v\} \otimes k[X] /\left(X^{2}-X\right)$ considered in [8, Theorem 4.4] is no longer an algebra map. Still, for these cases it is possible to consider the algebra isomorphism

$$
f: k\{u, v\} \otimes k[X] /\left(X^{2}-X\right) \longrightarrow \mathcal{M}_{2}(k)
$$

given by

$$
\begin{gathered}
u \longmapsto\left(\begin{array}{cc}
1 & 0 \\
0 & 0
\end{array}\right), \quad v \longmapsto\left(\begin{array}{ll}
0 & 0 \\
0 & 1
\end{array}\right), \\
u X v \longmapsto\left(\begin{array}{cc}
0 & a_{u} a_{v} \\
0 & 0
\end{array}\right), \quad v X u \longmapsto\left(\begin{array}{cc}
0 & 0 \\
a_{u} a_{v} & 0
\end{array}\right),
\end{gathered}
$$

in agreement with the result that we got in Proposition 3.1 .

Remark. It is worth noting that the fact that a matrix algebra may be written in this way shows that the twisted tensor product of two elementary algebras (as is the case for the algebras that we are considering) is not in general a elementary algebra, even if we require the twisting map to be bijective. Actually, the example that we present shows that we can build a twisted tensor product of two elementary algebras by means of an invertible twisting map, and obtain an algebra which is not even basic! 


\section{Hochschild COHOMOlOGY}

In this section we give a description of some facts related to the Hochschild cohomology of the twisted tensor product algebras that we have described above. Due to the similarity in the construction of the twisted tensor product with the one performed for the usual tensor product, it is reasonable to expect that Hochschild homology groups should satisfy a sort of (maybe twisted) Künneth formula that would allow to compute the homology groups of the twisted tensor product algebra out of the homology groups of the factors. A step in this direction was given by J. A. Guccione and J. J. Guccione in [9], where they build up a bicomplex which should allow to compute the (co)homology for the twisted tensor product when the twisting map is bijective, stating as a consequence that the Hochschild dimension of a twisted tensor product is bounded by the sum of the Hochschild dimensions of the factors. In particular, this result would imply that any twisted tensor product of two separable (i.e. having Hochschild dimension equal to 0) algebras is again separable.

This result is false, and the counterexample we consider shows that there is no hope to obtain a correct reformulation. We can build up a twisted tensor product of two separable algebras (both of them isomorphic to $k^{2}$ ) with respect to an invertible twisting map, and such that the resulting algebra does not even have finite Hochschild dimension. In order to do this, we give explicit descriptions, using some methods developed by Cibils in [7] and [8], of the Hochschild cohomology of all the algebras that we classified in the former section.

Proposition 4.1. Let $A \cong B \cong k^{2}$, and let $\tau: B \otimes A \rightarrow A \otimes B$ be a twisting map, then the Hochschild cohomology of twisted tensor product algebra $R:=A \otimes_{\tau} B$ is given by:

(I) If $R \cong k^{4}$, then $H H^{0}(R)=k^{4}$ and $H H^{n}(R)=0$ for any $n \geq 0$.

(IIa) If $R \cong \mathcal{M}_{2}(k)$, then $H H^{0}(R)=k$ and $H H^{n}(R)=0$ for any $n \geq 0$.

(IIb) If $R \cong k Q /\left(Q_{\geq 2}\right)$, then $H H^{n}(R)=k$ for all $n \geq 0$. In particular $R$ has infinite Hochschild dimension.

(III) If $R=k \widetilde{Q}$, then $H H^{0}(R)=k^{3}$, and $H H^{n}(R)=0$ for all $n \geq 1$.

Proof The cases $(I)$ and $(I I a)$ are trivial, since both $k^{4}$ and $\mathcal{M}_{2}(k)$ are separable algebras (the latest because it is Morita equivalent to the ground field $k$ ).

Case $(I I I)$ is a consequence of Theorem 1.2.

Case $(I I b)$ is a direct consequence of Proposition [1.3. Since this is the situation that provides us the aforementioned counterexample, for the sake of completeness, we sketch Cibil's procedure applied to this particular example:

Recall (cf. [5], [6]) that, if we have a finite dimensional algebra $R$ admitting a decomposition $R=E \oplus J$, being $E$ a maximal semisimple subalgebra of $R$ (which is separable) and $J$ the Jacobson radical of $R$, then the Hochschild cohomology of $R$ can be computed as the cohomology of the following complex of cochains:

$$
0 \rightarrow R^{E} \rightarrow \operatorname{Hom}_{E-E}(J, R) \rightarrow \cdots \cdots \rightarrow \operatorname{Hom}_{E-E}\left(J^{\otimes_{E}^{n}}, R\right) \rightarrow \cdots \cdots
$$


where $J^{\otimes_{E}^{n}}$ is the tensor product over $E$ of $n$ copies of $J$. Whenever the Jacobson radical satisfies that $J^{2}=0$, the coboundary is given by

$$
\begin{gathered}
(\delta r)(x):=r x-x r \quad \forall r \in R^{E}, x \in J \\
(\delta f)\left(x_{1} \otimes \cdots \otimes x_{n+1}\right)=x_{1} f\left(x_{2} \otimes \cdots \otimes x_{n+1}\right)+(-1)^{n+1} f\left(x_{1} \otimes \cdots \otimes x_{n}\right) x_{n+1}
\end{gathered}
$$

for all $f \in \operatorname{Hom}_{E-E}\left(J^{\otimes_{E}^{n}}, R\right)$. In our particular example, we have $k Q /\left(Q_{\geq 2}\right) \cong k Q_{0} \oplus k Q_{1}$, being $E=k Q_{0} \cong k^{2}$ the (commutative) maximal semisimple subalgebra of $R$ and $k Q_{1}=J$ its Jacobson radical (whose square is 0 ). It is immediate to check that $J^{\otimes_{E}^{n}}$ admits as a basis the set $Q_{n}$ of paths of length $n$. Now, using the additivity of the Hom functor, we have $\operatorname{Hom}_{E-E}\left(k Q_{n}, R\right) \cong \operatorname{Hom}_{E-E}\left(k Q_{n}, k Q_{0}\right) \oplus \operatorname{Hom}_{E-E}\left(k Q_{n}, k Q_{1}\right)$, and, as every simple subbimodule of $k Q_{n}$ corresponds to the bimodule generated by a path $\gamma$ of length $n$, which we can associate to the couple of vertices $(s(\gamma), t(\gamma))$ of starting and ending points of $\gamma$. Applying Schur's lemma, we have $\operatorname{Hom}_{E-E}\left(k \gamma, k \gamma^{\prime}\right)=0$ unless $\gamma$ and $\gamma^{\prime}$ have the same starting and ending points, that is, unless $\gamma$ and $\gamma^{\prime}$ are parallel paths. Using this, we find a linear isomorphism $\operatorname{Hom}_{E-E}\left(k Q_{n}, k Q_{0}\right) \simeq k\left(Q_{n} / / Q_{0}\right)$. Similarly, we have a linear isomorphism $\operatorname{Hom}_{E-E}\left(k Q_{n}, k Q_{1}\right) \simeq k\left(Q_{n} / / Q_{1}\right)$. Through these identifications, the coboundary $\delta$ is translated into the coboundary $\left(\begin{array}{ll}0 & 0 \\ D & 0\end{array}\right)$, where the map $D: k\left(Q_{n} / / Q_{0}\right) \rightarrow k\left(Q_{n+1} / / Q_{1}\right)$ is given by

$$
D(\gamma, e):=\sum_{a \in Q_{1} e}(a \gamma, \gamma)+(-1)^{n+1} \sum_{a \in e Q_{1}}(\gamma a, a) .
$$

By construction, we obtain a complex isomorphism between (4.10) and the complex $(4.11)$

$$
0 \rightarrow k Q_{0} \longrightarrow k\left(Q_{1} / / Q_{0}\right) \oplus k\left(Q_{1} / / Q_{1}\right) \longrightarrow \cdots \longrightarrow k\left(Q_{n} / / Q_{0}\right) \oplus k\left(Q_{n} / / Q_{1}\right) \longrightarrow \cdots .
$$

Since our quiver has no loops, whenever $n$ is odd we have

$$
k\left(Q_{n} / / Q_{0}\right)=k\left(Q_{n+1} / / Q_{1}\right)=\{0\},
$$

whilst for $n$ even we get

$$
k\left(Q_{n} / / Q_{0}\right) \cong k\left(Q_{n+1} / / Q_{1}\right) \cong k \mathbb{Z}_{2},
$$

as every path is uniquely determined by its starting (and ending) point, where the identification consists on sending a path to 1 if it starts at the vertex $e$ or to $t$ if it starts at the vertex $f$, and we are considering $k \mathbb{Z}_{2}=k\left\{1, t \mid t^{2}=1\right\}$. Via this identification, for even $n$, the map $D$ transforms into the map $D^{\prime}: k \mathbb{Z}_{2} \rightarrow k \mathbb{Z}_{2}$ defined by

$$
D^{\prime}(1)=1-t, D^{\prime}(t)=t-1 \text {. }
$$

This map obviously has one dimensional kernel, generated by the element $1+t$, and one dimensional image. Summing everything up, we may rewrite the complex (4.11) as

$$
0 \longrightarrow k^{2} \stackrel{D^{\prime}}{\longrightarrow} k^{2} \stackrel{0}{\longrightarrow} k^{2} \stackrel{D^{\prime}}{\longrightarrow} \cdots \cdots \cdots
$$

and thus, for $n$ odd we have

$$
\operatorname{dim}_{k} H H^{n}\left((k Q)_{2}\right)=\operatorname{dim}_{k}\left(\frac{\operatorname{ker} 0}{\operatorname{Im} D^{\prime}}\right)=\operatorname{dim}_{k} k^{2}-\operatorname{dim}_{k}\left(\operatorname{Im} D^{\prime}\right)=1,
$$


whilst, for $n$ even we get

$$
\operatorname{dim}_{k} H H^{n}\left((k Q)_{2}\right)=\operatorname{dim}_{k}\left(\frac{\operatorname{ker} D^{\prime}}{\operatorname{Im} 0}\right)=\operatorname{dim}_{k}\left(\operatorname{ker} D^{\prime}\right)-\operatorname{dim}_{k}(0)=1,
$$

as we wanted to prove.

As we announced, the algebra of type $(I I b)$ provides us an example of a twisted tensor product of two separable algebras, with respect to a bijective twisting map, which does not have finite Hochschild dimension. This example contradicts [9, Theorem 1.6, Theorem 1.7, Corollary 1.8]. It is worth noting that in order to disprove Gucciones' results, it is not necessary to give an explicit description of the Hochschild cohomology, being enough to show that the twisted tensor product algebra is not separable. An immediate prove of this fact follows from the realization of this algebra as the quotient $R=k Q /\left(Q_{\geq 2}\right)$, as we can immediately check that the elements of $R$ corresponding to (the equivalence classes of) the arrows of $Q$ provide nonzero elements of the Jacobson radical of $R$ (actually, the Jacobson radical is precisely the ideal generated by these two elements).

\section{ACKNOWLEDGEMENTS}

The authors would like to thank Dragos Stefan for helping us to understand the subtleties beyond Hochschild cohomology, and Calude Cibils, for his willingness to check our work and his numerous remarks and corrections to the preliminary version of this paper.

\section{REFERENCES}

[1] J. Beck, Distributive laws, Lecture Notes in Mathematics 80 (1969), 119-140.

[2] S. Caenepeel, B. Ion, G. Militaru and S. Zhu, The factorization problem and the smash biproduct of algebras and coalgebras, Algebr. Represent. Theory 3 (2000), no. 1, 19-42.

[3] A. Cap, H. Schichl and J. Vanzura, On twisted tensor products of algebras, Comm. Algebra, 23 (12), (1995), 4701-4735.

[4] P. Cartier, Produits tensorieles tordus, Exposé au Seminaire des groupes quantiques de l'Ecole Normale Supérieur, Paris 1991-1992.

[5] C. Cibils, Cyclic and Hochschild homology of 2-nilpotent algebras. K-Theory 4 131-141 (1990).

[6] C. Cibils, Rigidity of truncated quiver algebras. Advances in Math. 79 18-42 (1990).

[7] C. Cibils, Hochschild cohomology algebra of radical square zero algebras, Algebras and modules, II (Geiranger, 1996), 93-101, CMS Conf. Proc. 24, Amer. Math. Soc, Providence, RI, 1998.

[8] C. Cibils, Non-commutive duplicates of finite sets, J. Algebra Appl. 5 (2006), no. 3, 361-377.

[9] J. A. Guccione and J. J. Guccione, Hochschild homology of twisted tensor products, K-Theory 18 (1999), no. 4, 363-400.

[10] P. Jara Martínez, J. López Peña, F. Panaite, and F. Van Oystaeyen, On iterated twisted tensor products of algebras, math.QA/0511280

[11] P. Jara Martínez, J. López Peña, D. Stefan, On Koszulity and Hochschild (co)homology of twisted tensor products, In preparation.

[12] S. Majid. Physics for algebraists: Non-commutative and non-cocommutative Hopf algebras by a bicrossproduct construction. J. Algebra, 130:17-64, 1990. 
Department of Algebra, University of Granada, Avda. Fuentenueva s/n, E-18071, Granada, SPAin

E-mail address: jlopez@ugr.es, gnavarro@ugr.es 